\title{
La vaccination contre le SRAS-CoV-2 devrait être obligatoire pour exercer la médecine au Canada
}

\author{
Andrew D. McRae MD PhD, Andreas Laupacis MD MSc
}

Citation : CMAJ 2021 November 29;193:E1816-7. doi : 10.1503/cmaj.211839-f; diffusion hâtive le 9 novembre 2021

Voir la version anglaise de l'article ici : www.cmaj.ca/lookup/doi/10.1503/cmaj.211839

$\mathbf{L}$ es centres hospitaliers et les autorités sanitaires du Canada exigent la vaccination contre le SRAS-CoV-2 pour leurs médecins et les membres de leur personnel. La vaste majorité des médecins canadiens se sont fait vacciner contre le SRAS-CoV-2 et plusieurs autorités sanitaires exigent que les travailleurs de la santé soient vaccinés. Cependant, quelques médecins ont protesté contre la vaccination obligatoire en lien avec le SRAS-CoV-2 chez les travailleurs de la santé, certains annonçant publiquement leur décision de ne pas se faire vacciner et intentant des poursuites contre les autorités sanitaires ${ }^{1}$. Ces médecins ébranlent la confiance du public envers l'innocuité et l'efficacité des vaccins contre le SRAS-CoV-2 et mettent à risque les patients et les collègues du milieu de la santé. Les ministères de la Santé provinciaux devraient exiger la vaccination obligatoire des travailleurs de la santé. Les médecins qui refusent la vaccination contre le SRAS-CoV-2 et qui n'ont pas d'exemption médicale valide devraient se voir interdire l'exercice de la médecine en personne.

Certains médecins qui ont choisi de ne pas se faire vacciner ont interpellé les autorités sanitaires et les ordres professionnels, soutenant que les vaccins contre le SRAS-CoV-2 en sont encore au stade expérimental et qu'ils sont inefficaces et dangereux. Des données probantes réfutant ces allégations existent. Les résultats d'essais cliniques randomisés et contrôlés, réalisés avant l'approbation des vaccins et comptant plus de 70000 participants, ont démontré l'efficacité de la vaccination à diminuer l'infection et la prévalence des formes graves de la COVID-192,3. Des preuves convaincantes tirées des données de suivi des millions de doses administrées jusqu'à maintenant révèlent que le vaccin est efficace et que les événements indésirables graves sont à la fois rares ${ }^{4}$ et moins fréquents que les risques associés à la forme grave de la COVID-19 chez les personnes non vaccinées ${ }^{5,6}$.

L'argument le plus important pour la vaccination universelle des médecins est la prévention de la transmission du SRAS-CoV-2 par un médecin infecté à ses patients et ses collègues. Avec l'émergence du variant Delta, lequel est plus virulent et transmissible que les variants précédents ${ }^{5,7}$, la vaccination universelle des travailleurs de la santé est encore plus importante afin de prévenir la transmission du SRAS-CoV-2 aux patients et entre travailleurs de la santé.

Cette vaccination universelle représente une protection additionnelle pour les patients, au-delà de l'utilisation d'équipements de protection individuelle. Bien que plusieurs patients hospitalisés à l'heure actuelle soient vaccinés contre le SRAS-CoV-2, certains ne le sont pas. Certains patients vaccinés sont immunodéprimés, fragiles ou présentent des comorbidités qui les mettent à risque de présenter une immunité décroissante ou de subir de graves répercussions de la COVID-19.

Pour les médecins, l'obligation vaccinale de réduire le risque d'infection nosocomiale concorde avec leurs obligations fiduciaires envers leurs patients. Elle est soutenue par des principes éthiques de bienfaisance et de non-malfaisance, qui se traduisent par l'obligation de prodiguer des soins efficaces et sécuritaires. Il existe des précédents de politiques de vaccination obligatoire des travailleurs de la santé ayant pour but de diminuer les risques posés aux patients. Plusieurs établissements de soins de santé exigent que les médecins et les membres du personnel soient vaccinés contre la grippe ${ }^{8}$ et l'immunisation contre la rubéole est exigée pour toutes les personnes qui prodiguent des soins aux patients dans les établissements hospitaliers de l'Alberta et de l'Ontario ${ }^{9,10}$. De tels mandats préservent aussi la capacité des employés de la santé en atténuant le risque d'infections contractées dans la collectivité et en diminuant le risque de transmission en milieu de travail parmi les travailleurs de la santé. Au cours de la première année de la pandémie, les infections professionnelles chez les travailleurs de la santé étaient souvent causées par une transmission entre membres du personnel ${ }^{11}$. Le principe éthique de justice, dont est issue l'obligation d'assurer des soins sécuritaires et efficaces, soutient la vaccination obligatoire afin de maintenir des effectifs 
de soins de santé fonctionnels en limitant le risque de transmission du SRAS-CoV-2 entre les travailleurs de la santé.

Les médecins qui s'opposent à la vaccination contre le SRASCoV-2 prétendent qu'ils ont la liberté de choix face au vaccin tout en poursuivant l'exercice de leur pratique clinique. Puisque le choix de ne pas se faire vacciner pose un risque pour les patients et les collègues, ce choix entre en conflit avec les obligations professionnelles d'un médecin. Si ce dernier choisit de ne pas être vacciné contre le SRAS-CoV-2, il est raisonnable, voire nécessaire, que les autorités hospitalières et réglementaires restreignent ses activités professionnelles.

Les obligations vaccinales sont des mesures exceptionnelles : elles limitent le libre choix individuel en matière de vaccination de façon plus importante que ce qui est habituellement accepté. Mais le SRAS-CoV-2 demeure une menace exceptionnelle pour les patients et la population. Les responsabilités professionnelles des médecins de prodiguer des soins sécuritaires et d'assurer le maintien du système de soins de santé sont issues de principes éthiques reconnus, ont préséance sur ce qui pourrait habituellement être un choix personnel. Les personnes qui résistent à la vaccination sont libres de refuser les vaccins. Mais elles ne devraient pas être libres de refuser la vaccination contre le SRAS-CoV-2 tout en exerçant comme médecin.

\section{Références}

1. Gibson J. 4 Alberta doctors launch lawsuit over mandatory COVID-19 vaccine policy. CBC News updated 2021 Oct. 26. Accessible ici : https://www.cbc.ca/news/canada/ calgary/ahs-lawsuit-vaccine-mandatory-calgary-1.6224278 (consulté le 27 oct. 2021).

2. Regulatory Decision Summary. Comirnaty. Ottawa: Health Canada; 2021. Accessible ici : https://covid-vaccine.canada.ca/info/regulatory-decision-summary -detail.html?linkID=RDS00856 (consulté le 27 oct. 2021).

3. Regulatory Decision Summary. Spikevax. Ottawa: Health Canada; 2021. Accessible ici : https://covid-vaccine.canada.ca/info/regulatory-decision-summary -detail.html?linkID=RDS00855 (consulté le 27 oct. 2021).

4. Witberg G, Barda N, Hoss S, et al. Myocarditis after COVID-19 vaccination in a large health care organization. N Engl J Med 2021 Oct. 6 [Cyberpublication avant impression]. doi : 10.1056/NEJMoa2110737.

5. Fisman DN, Tuite AR. Evaluation of the relative virulence of novel SARS-CoV-2 variants: a retrospective cohort study in Ontario, Canada.CMAJ 2021;193:E1619-25.

6. Chung H, He S, Nasreen S, et al. Effectiveness of BNT162b2 and mRNA-1273 COVID-19 vaccines against symptomatic SARS-CoV-2 infection and severe COVID19 outcomes in Ontario, Canada: test negative design study. BMJ 2021;374:n1943.

7. Scientific brief: SARS-CoV-2 transmission. Atlanta: Centers for Disease Control and Prevention; 2021. Accessible ici : https://www.cdc.gov/coronavirus/2019-ncov/ science/science-briefs/sars-cov-2-transmission.html (consulté le 27 oct. 2021).

8. Flood CM, Thomas B, Wilson K. Mandatory vaccination for health care workers: an analysis of law and policy. CMAJ 2021;193:E217-20.

9. Communicable Diseases Regulation, Alta Reg 238/1985. Public Health Act.

10. Rubella surveillance protocol for Ontario hospitals. Toronto: Ontario Hospital Association; reviewed and revised 2019. Accessible ici : https://www.oha.com/ Documents/Rubella\%20Protocol\%20(May\%202019).pdf (consulté le 28 oct. 2021).

11. Sikkens JJ, Buis DTP, Peters EJG, et al. Serologic surveillance and phylogenetic analysis of SARS-CoV-2 infection among hospital health care workers. JAMA Netw Open 2021;4:e2118554.

\section{Intérêts concurrents : www.cmaj.ca/staff}

Affiliations : Boursier en rédaction, JAMC (McRae); Départements de médecine d'urgence et des sciences de la santé communautaire (McRae), Université de Calgary, Calgary, Alb.; rédacteur adjoint principal, JAMC (Laupacis)

Propriété intellectuelle du contenu : Il s'agit d'un article en libre accès distribué conformément aux modalités de la licence Creative Commons
Attribution (CC BY-NC-ND 4.0), qui permet l'utilisation, la diffusion et la reproduction de tout médium à la condition que la publication originale soit adéquatement citée, que l'utilisation se fasse à des fins non commerciales (c.-à-d., recherche ou éducation) et qu'aucune modification ni adaptation n'y soit apportée. Voir : https://creativecommons.org/ licenses/by-nc-nd/4.0/deed.fr.

Correspondance : Rédaction du JAMC, editorial@cmaj.ca 